POS PROCEEDINGS

\title{
The Local Bubble as a cosmic-ray isotropizer
}

\author{
Iris Gebauer* \\ Karlsruhe Institute of Technology, Institut für Experimentelle Kernphysik, D-76131 Karlsruhe, \\ Germany \\ E-mail: gebauerekit.edu
}

\section{Matthias Weinreuter}

Karlsruhe Institute of Technology, Institut für Experimentelle Kernphysik, D-76131 Karlsruhe, Germany

\section{Simon Kunz}

Karlsruhe Institute of Technology, Institut für Experimentelle Kernphysik, D-76131 Karlsruhe, Germany

\section{Daniele Gaggero}

SISSA, via Bonomea 265, I-34136, Trieste, Italy

INFN, sezione di Trieste, via Valerio 2, I-34127, Trieste, Italy

\begin{abstract}
Recently, the PAMELA, Fermi-LAT and AMS-02 collaborations have measured an anomalous population of high-energy positrons, not compatible with the conventional picture of secondary production by the collision of cosmic ray nuclides with the interstellar medium.

Two possible origins for this antiparticle population have been extensively discussed in the literature: astrophysical point sources (e.g. local pulsars), and dark matter annihilation/decay.

In the first case a non negligible anisotropy in the flux is expected, possibly pointing towards a nearby pulsar.

In this work we show that any reliable prediction of the anisotropy level for leptons needs to account for the peculiar environment surrounding the Solar System.

In particular, we discuss the role of the so-called Local Bubble, an underdense region, embedded in a dense wall of molecular clouds: This structure is expected to act as an efficient cosmicray isotropizer. Using realistic assumptions on the impact of the Local Bubble on cosmic-ray diffusion, we demonstrate that the Local Bubble can indeed dilute the directional information of energetic positrons and electrons.

We also show the impact of the bubble on hadronic and leptonic spectra measured at Earth.
\end{abstract}

The 34th International Cosmic Ray Conference,

30 July- 6 August, 2015

The Hague, The Netherlands

\footnotetext{
* Speaker.
} 


\section{Introduction}

We examine in this work the impact of local structures on the leptonic and hadronic component of cosmic rays (CRs). In particular, we discuss how these structures change the predictions of numerical models regarding both spectra and arrival directions.

It is well known that the transport of electrons and positrons is characterized by severe energy losses due to synchrotron emission in the galactic magnetic field and Inverse Compton scattering on the interstellar radiation field. For this reason their propagation length decreases significantly with increasing energy from values of around $10 \mathrm{kpc}$ at $\simeq 1 \mathrm{GeV}$ to $600-900 \mathrm{pc}$ at $\simeq 1 \mathrm{TeV}$. With propagation distances as low as a few hundred parsecs, the structures in the local interstellar medium (ISM) are therefore expected to have a sizable effect on the local spectra and arrival directions of the CR leptons.

Depending on the propagation setup, also hadrons are expected to be quite local, especially at high energy, due to the vertical escape out of the diffusion halo [1]. Moreover, compact structures with very different properties (e.g. much more turbulence) with respect to the Galactic average may alter this component as well.

The most prominent structure in our local ISM is the so-called Local Bubble, an underdense region containing our Solar system close to its center, and surrounded by a wall of dense molecular clouds. The shape of the bubble is asymmetric and similar to a chimney, elongated perpendicularly to the Galactic plane and opened towards the halo, with radii ranging between $\simeq 50 \mathrm{pc}$ and $\simeq 150$ pc (see e.g. [2], [3]).

The Local Bubble is connected via low-density tunnels to other similar structures [4]. These huge connected cavities filled with hot ionized gas are expected to originate from supernova explosions and should be present in every region of the Galaxy, making the Galactic disk very inhomogeneous due to the presence of many underdense regions surrounded by denser and colder molecular clouds.

The regions surrounding these bubbles are highly magnetized and turbulent and therefore should confine CRs very efficiently; since the CR diffusion coefficient is expected to be strongly dependent on the ISM properties (see e.g. [5]), a variation of more than one order of magnitude passing from the underdense bubbles to the surrounding regions is allowed, and - as we will show below - such a strong fluctuation has a huge impact on high-energy lepton and hadron propagation, changing in a relevant way the predictions regarding local spectra and anisotropy. In particular, the intuitive idea is that the efficient confinement exptected in the highly turbulent regions surrounding the bubble should trap CRs for a longer time (compared to standard predictions), in such a way that the anisotropy expected from a local source just outside the bubble is decreased.

The local anisotropy in leptonic fluxes is particularly important in order to understand the so-called positron excess recently reported by PAMELA, Fermi-LAT and AMS-02 experiments $[6,7,8]$ : Indeed, a positive detection of a dipole anisotropy towards a known object (e.g. a pulsar) could prove the astrophysical origin of the anomaly (see e.g. $[9,10]$ and references therein).

No detection of any anisotropy has been reported so far, but more and more stringent upper limits are being placed on the total flux of electrons and positrons and on the separate positron flux.

In this context, the isotropizing effect due to the bubble could help to explain a possible tension between model predictions and upper limits. 


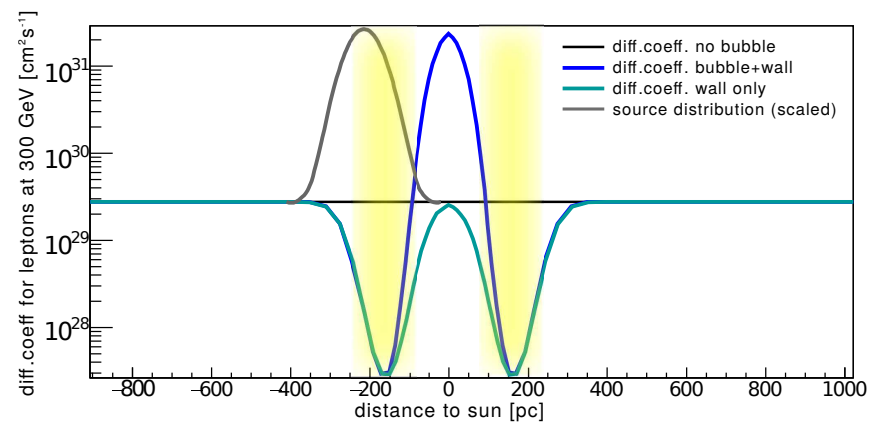

Figure 1: The behavior of the diffusion coefficient in the Local Bubble for the two scenarios considered here. The gray line shows the pulsar source distribution.

\section{Model}

We use the DRAGON ${ }^{1}$ code to simulate the propagation of $e^{ \pm}$accelerated by a nearby point source through the environment of the Local Bubble.

This package is designed to solve numerically the transport equation for all CR species in both 2D and 3D mode, featuring both isotropic and anisotropic diffusion; the 3D simulations can be performed with non-equidistant binning in order to resolve small local structures with sufficient accuracy (in our case, e.g., the spatial resolution of our model around the position of the Sun is as low as $10 \mathrm{pc}$ ). The code includes the nuclear cross section database and interstellar radiation field model from the Galprop code $[13,15,14]$.

The transport model is designed to describe the locally measured proton and antiproton spectrum, the $\mathrm{B} / \mathrm{C}$ ratio and the ${ }^{10} \mathrm{Be} /{ }^{9} \mathrm{Be}$ fraction. More precisely, it is chosen among a set of models considered in the detailed MCMC analysis presented in [17] and compatible with several sets of pre-AMS official data within $1 \sigma$. The model does not feature any significant convection or reacceleration, and is characterized by a power-law rigidity scaling of the diffusion coefficient with index $\delta=0.57$.

Using this setup, we compute the contribution of secondary $e^{ \pm}$from proton-gas interactions in the ISM. The solution is obtained separately for continuously distributed supernova remnants (where primary CR acceleration takes place for both leptons and hadrons; the latter component also produces secondary positrons and electrons in subsequent interactions with the ISM), and for a pulsar point source (considered as electron-positron pair emitter).

- We model the source as a Gaussian with a width of $60 \mathrm{pc}$ in order to account for the extension of the pulsar wind nebula; the source is assumed to be located at a distance $d=200$ pc toward the Galactic center, just outside the bubble wall. The common electron and positron spectrum from pulsars is assumed to follow a power law with spectral index $\gamma$ and an exponential cutoff at energy $E_{\text {cut }}: Q_{e^{+}, e^{-}}^{\text {pulsar }}=q_{0}\left(E / E_{0}\right)^{-\gamma} \exp \left(-E / E_{\text {cut }}\right)$. The position and extension of the source with respect to the Local Bubble is illustrated by the gray line in Fig.1. The source parameters are fixed to best describe the AMS-02 positron data.

\footnotetext{
${ }^{1}$ the code is public and available at www. dragonproject. org $[11,12]$
} 
- To model the Local Bubble we implement a region with increased spatial resolution surrounding the Sun in the DRAGON code. Within this region the normalization of the diffusion coefficient is allowed to vary as described in the following.

In the most realistic model the bubble is a region of large diffusion coefficient surrounded by a thin, very confining region characterized instead by a low diffusion coefficient. The diffusion coefficient has the lowest value at a distance of $160 \mathrm{pc}$ from the Sun. We discuss three scenarios.

- A decrease of diffusion coefficient in the bubble wall by a factor of 10 with respect to the galactic value, and an increase by a factor of 10 with respect to the galactic value at the Sun's position.

- A decrease of diffusion coefficient in the bubble wall by a factor of 100 with respect to the galactic value, and an increase by a factor of 100 with respect to the galactic value at the Sun's position (Strong wall + bubble scenario: dark blue line in Fig.1)

- A decrease of diffusion coefficient in the bubble wall by a factor of 100 with respect to the galactic value, while the diffusion coefficient at the position of the Sun is identical to the galactic value (Strong wall scenario: sea green line in Fig.1).

More details about the technical implementation of the Local Bubble will be presented in a forthcoming publication.

For each bubble scenario the point source parameters are optimized to best describe the AMS02 positron and electron data, as can be seen in Fig. 2.

\section{Impact on anisotropy and spectra}

The CR anisotropies are numerically calculated for the position of the Sun according to $\delta=$ $3 D(E) / c \cdot \nabla n_{\mathrm{e}} / n_{\mathrm{e}}$, where $D(E)$ is the local diffusion coefficient and $n_{e}$ is the local electron density. The spatial resolution at the position of the Sun in $10 \mathrm{pc}$.

We show in Fig. 3 the anisotropy of the electron and positron sum flux. The standard scenario with no Local Bubble yields anisotropies at the level of $\sim 1 \%$ at $\simeq 100 \mathrm{GeV}$, very near the $95 \% \mathrm{CL}$ upper limits from Fermi-LAT.

In the strong wall + bubble scenario the level of anisotropy is decreased by a factor of $\simeq 2$ in the entire energy range, while the strong wall scenario yields a decrease by a factor of about 4 .

The plot clearly shows that, in order to have a significant isotropizing effect, the change in the diffusion coefficient between the inner part of the bubble and the wall must be very large, since only the strong scenarios characterized by a factor of 100 between inner and outer diffusion coefficient lead to an efficient isotropization of the flux. As discussed at the beginning, given the very different properties of the ISM in the inner part of the bubble and in the surrounding clouds, such a strong fluctuation in the transport properties is acceptable.

The impact of these scenarios on the CR spectra for both leptonic and hadronic species is shown in Fig. $4-7$. The plots show that, in the weak scenario, the impact on the observables we considered is below the percent level in almost all cases. In the more interesting strong scenario, 


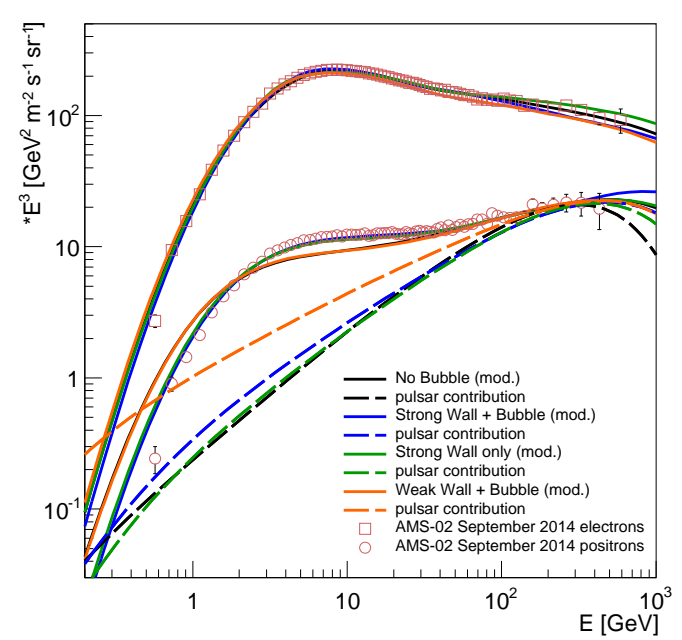

Figure 2: Electron and positron spectra measured by AMS-02 compared to our model. For each bubble scenario the pulsar parameters have been optimized for the data.

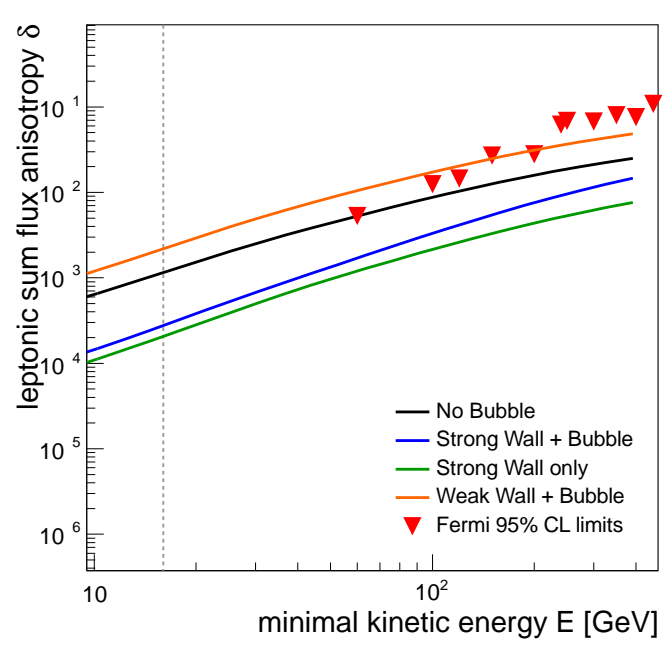

Figure 3: Electron+positron sum flux anisotropy. The $\mathrm{x}$-axis is in cumulative energies, with a maximum energy of $1 \mathrm{TeV}$. Data: Fermi-LAT [16].

where the isotropizing effect we want to discuss is large, the impact on CR spectra is quite large, due to the large confinement time within the wall, with subsequent increased secondary production and alterations in the energy losses. In particular, we point out a $40 \%$ change in the high-energy $\mathrm{B} / \mathrm{C}$ ratio above $100 \mathrm{GeV}$. For this reason, the propagation models need to be retuned when this effect is taken into account. We pospone to a forthcoming work a more detailed discussion on the consequences arising from the presence of a Local Bubble modeled in this way on our knowledge of the Galactic CR transport parameters. Furthermore, in this study we constrained ourselves to variations in the diffusion coefficient which are associated to variations in the insterstellar medium. The corresponding changes in the local gas density do not influence the CR arrival directions and have been neglected here. Naturally, changes in the local gas density, specifically in regions with comparably low diffusion coefficient, increase the local CR interaction rate and the energy losses. As an example, assuming an increase in gas density by a factor of 5 in the bubble wall leads to an additional increase of about $10 \%$ in the boron to carbon ratio at $\mathrm{GeV}$ energies, due to increased secondary production in the dense material, and a decrease of about $50 \%$ below $0.1 \mathrm{GeV}$, due to increased energy losses.

\section{Conclusion}

We demonstrated that the Local Bubble can indeed act as an efficient isotropizer for leptonic CR incoming directions. The level of anisotropy expected for a single $e^{ \pm}$point source can be decreased by a factor of 3 in the most efficient scenario considered here. The observed decrease in anisotropy crucially depends on the assumptions made about the local behaviour of the diffusion coefficient. It is noteworthy, that in the weak bubble scenario an increase in anisotropy is observed. 
positrons, relative to 'No Bubble' (with solar modulation)

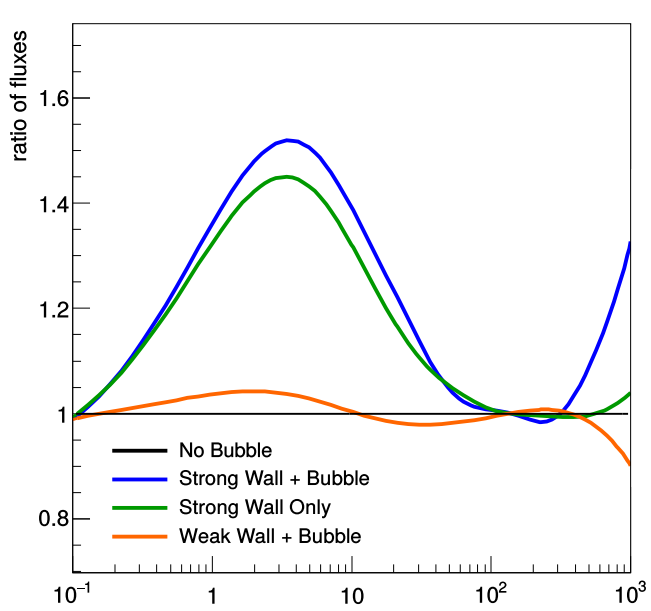

Figure 4: Impact of the bubble on positron spectrum.

protons, relative to 'No Bubble' (no solar modulation)

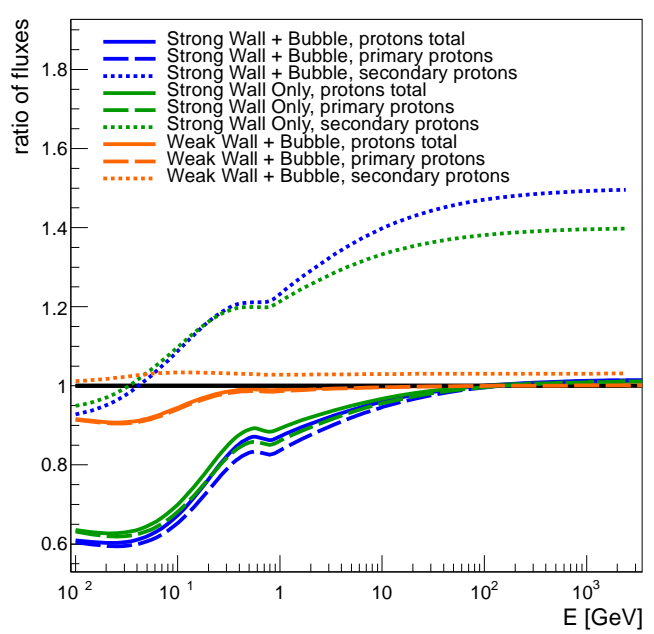

Figure 6: Impact of the bubble on proton spectrum. The full line corresponds to the total proton flux, while the long dashed line refers to the primary protons and the dotted line refers to the secondary protons.
B/C, relative to 'No Bubble' (no solar modulation)

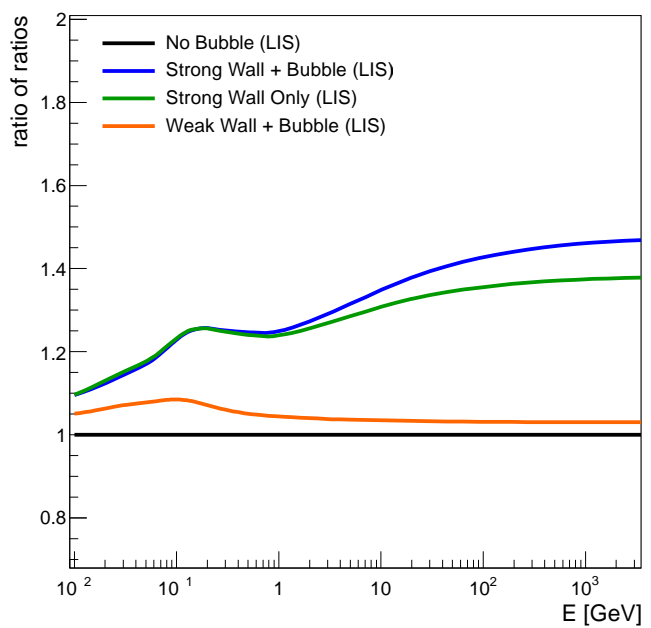

Figure 5: Impact of the bubble on $\mathrm{B} / \mathrm{C}$ spectrum.

$\mathrm{Be}^{10} / \mathrm{Be}^{9}$, relative to 'No Bubble' (no solar modulation)

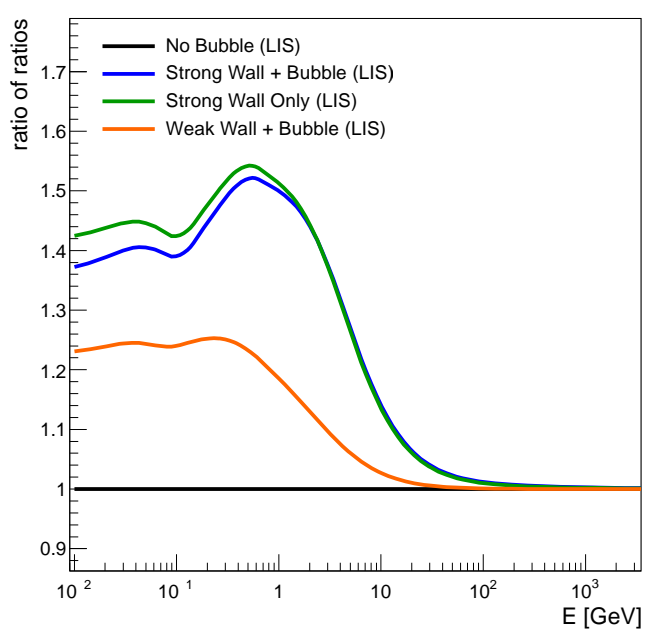

Figure 7: Impact of the bubble on Be spectrum.

We also showed that local variations in the diffusion coefficient can significantly change the shape of local CR spectra. While the changes in the electron and positron spectra can be compensated by a suitable adaptation of the injection spectra of primary electrons and positrons (the first originating from conventional CR accelerators like supernova remnant and the assumed local point source, the latter originating exclusively from the assumed local $e^{ \pm}$point source), the observed changes in the local spectra of nuclei and secondary to primary ratios require a retuning of the propagation model. 
In this study we assumed the global transport parameters of our model to be representative of the galactic average. Structures like the Local Bubble are expected to be present in the entire Galactic disk, leading to fluctuations in CR density and in CR interaction rates. For the strong bubble scenario we observe fluctuations in the proton density of up to $50 \%$ in the $\mathrm{GeV}$ and $\mathrm{TeV}$ range, while the local boron to carbon ratio, a measure of the $\mathrm{CR}$ interaction rate, is increased by about $40 \%$. We note that these fluctuations may have a significant impact on the diffuse galactic $\gamma$-ray emission origination from neutral pions produced primarily in proton gas interactions. In fact, studies of the diffuse galactic emission in the GeV range show that factors of up to $100 \%$ are required to describe the data $[17,18]$.

\section{References}

[1] C. Evoli, I. Cholis, D. Grasso, L. Maccione and P. Ullio, Phys. Rev. D 85, 123511 (2012) [arXiv:1108.0664 [astro-ph.HE]].

[2] H. A. Abt, American Institute of Physics Conference Series, 1452, 9 (2012)

[3] B. Y Welsh, D. M. Sfeir, M. M. Sirk, \& R. Lallement, Astronomy and Astrophysics, 352, 308 (1999)

[4] P.P. Plucinsky, American Institute of Physics Conference Series, 1156, 231 (2009)

[5] D. De Marco, P. Blasi and T. Stanev, JCAP 0706, 027 (2007) [arXiv:0705.1972 [astro-ph]].

[6] L. Accardo et al. [AMS Collaboration], Phys. Rev. Lett. 113, 121101 (2014).

[7] M. Ackermann et al. [Fermi-LAT Collaboration], Phys. Rev. Lett. 108, 011103 (2012) [arXiv:1109.0521 [astro-ph.HE]].

[8] O. Adriani et al. [PAMELA Collaboration], Phys. Rev. Lett. 111, 081102 (2013) [arXiv:1308.0133 [astro-ph.HE]].

[9] D. Grasso, S. Profumo, A. W. Strong, L. Baldini, R. Bellazzini, E. D. Bloom, J. Bregeon and G. Di Bernardo et al., Nucl. Instrum. Meth. A 630, 48 (2011).

[10] G. Di Bernardo, C. Evoli, D. Gaggero, D. Grasso, L. Maccione and M. N. Mazziotta, Astropart. Phys. 34, 528 (2011) [arXiv:1010.0174 [astro-ph.HE]].

[11] C. Evoli, D. Gaggero, D. Grasso and L. Maccione, JCAP 0810 (2008) 018 [arXiv:0807.4730 [astro-ph]].

[12] D. Gaggero, D. Grasso, L. Maccione, G. Di Bernardo and C. Evoli, Phys. Rev. D 89, 083007 (2014) [arXiv:1311.5575 [astro-ph.HE]].

[13] http://galprop.stanford.edu/

[14] T. A. Porter and A. W. Strong, astro-ph/0507119.

[15] A.W. Strong, I.V. Moskalenko, and V.S. Ptuskin., Ann.Rev.Nucl.Part.Sci., 57:285âĂŞ327 (2007)

[16] M. Ackermann et al. [Fermi-LAT Collaboration], Phys. Rev. D 82, 092003 (2010) [arXiv:1008.5119 [astro-ph.HE]].

[17] S. Kunz: Constraints on Transport Models for Galactic Cosmic Rays and their Implications for the Anomalous Positron Abundance, Phd thesis, KIT,

https://ekp-invenio.physik. uni-karlsruhe. de/record/ 48605 (2014)

[18] W. de Boer, I. Gebauer, and S. Kunz, Evidence for a hadronic origin of the Fermi Bubbles, formed by outflows from star-forming regions, this volume. 